\title{
Avaliação de interpoladores para o estudo de vulnerabilidade aquífera: $O$ caso da Bacia do Rio Santa Maria
}

\section{Evaluation of interpolators for aquifer vulnerability study: The case of the Santa Maria River Basin}

\author{
Thiago Boeno Patricio Luiz ${ }^{*}$, Leônidas Luiz Volcato Descovi Filho², José Luiz Silvério da Silva ${ }^{3}$
}

\begin{abstract}
Resumo: Estudos de avaliação da vulnerabilidade natural de aquíferos são de relevante importância para o conhecimento de áreas sujeitas à contaminação ambiental. A correta aplicação em mapas de vulnerabilidade necessita de estudos constantes, visando o aperfeiçoamento das análises e o desenvolvimento de mapas representativos das áreas estudadas. Objetivou-se, neste contexto, avaliar o desempenho de diferentes modelos para a interpolação de indicadores ambientais de vulnerabilidade aquífera, utilizando a técnica de krigagem. O estudo procedeu-se na Bacia Hidrográfica do Rio Santa Maria por meio da análise dos aspectos hidrogeológicos de 255 poços tubulares, localizados em seis municípios no sudoeste do Estado do Rio Grande do Sul. Os dados indicaram que a classe de alta vulnerabilidade predominou em 47,45\% dos poços avaliados, seguida da classe de média vulnerabilidade correspondendo a 23,14\%; a baixa vulnerabilidade representou 14,50\%, a classe de extrema vulnerabilidade aquífera correspondeu a 7,85\% e a insignificante indicou 7,05\%. Para interpolação dos dados, foram avaliados os modelos esférico, exponencial, gaussiano e linear, com o intuito de definir o melhor ajuste a partir da aplicação da função de semivariograma. Os ajustes foram avaliados através do método dos mínimos quadrados, que evidenciou a melhor abordagem a partir do modelo esférico dentre os modelos testados. A distribuição espacial da vulnerabilidade demonstrou o predomínio de alta vulnerabilidade na porção sul do município de Santana do Livramento, porção central de Rosário do Sul, norte e noroeste de São Gabriel e centro de Lavras do Sul. Em algumas áreas da bacia, nos municípios de Santana do Livramento e Lavras do Sul foi constatada a classe de extrema vulnerabilidade aquífera.
\end{abstract}

Palavras-chave: Água subterrânea; Contaminação; Semivariograma; Krigagem.

\begin{abstract}
Studies evaluating the natural vulnerability of aquifers have relevant importance for the knowledge of subject environmental contamination areas. The correct application in vulnerability maps needs constant studies, aiming the improvement of the analyzes and the development of representative maps of the studied areas. The objective of this study was to evaluate the performance of different models for the interpolation of environmental indicators of aquifer vulnerability using the kriging technique. The study was carried out in the Santa Maria River Basin by analyzing the hydrogeological aspects of 255 tube wells, located in six municipalities in the southwest of the State of Rio Grande do Sul. The data indicated that the high vulnerability class prevailed in $47,45 \%$ of the evaluated wells, followed by the medium vulnerability class corresponding to $23,14 \%$; the low vulnerability represented $14,50 \%$, the extreme vulnerability class corresponded to $7,85 \%$ and insignificant indicated 7,05\%. The spherical, exponential, gaussian and linear models were used to interpolate the data, to define the best fit from the application of the semivariogram function. The adjustments were evaluated through the least squares method, which evidenced the best approach from the spherical model among the tested models. The spatial distribution of vulnerability demonstrated the predominance of high vulnerability in the southern portion of the municipality of Santana do Livramento, central portion of Rosário do Sul, north and northeast of São Gabriel and center of Lavras do Sul. In some areas of the basin, in municipalities of Santana do Livramento and Lavras do Sul was found the class of extreme vulnerability aquifer.
\end{abstract}

Key words: Groundwater; Contamination; Semivariogram; Kriging.

\footnotetext{
*Autor para correspondência

Recebido para publicação em 04/07/2017; aprovado em 30/09/2017

${ }^{1}$ Engenheiro Sanitarista e Ambiental e Mestre em Engenharia Civil, Universidade Federal de Santa Maria, Santa Maria. Fone: (51) 99227-3370, E-mail: thiagoboeno@hotmail.com.

${ }^{2}$ Doutor em Geografia. Professor do Instituto de Engenharia e Geociências da Universidade Federal do Oeste do Pará. E-mail: leonprs@ gmail.com

${ }^{3}$ Doutor em Geologia. Professor titular da Universidade Federal de Santa Maria. E-mail: silverioufsm@ gmail.com
} 


\section{INTRODUÇÃO}

O estudo de vulnerabilidade aquífera representa uma importante ferramenta para definir as áreas que são naturalmente mais vulneráveis à poluição. Associada aos pontos potenciais de contaminação e pelo tipo do ambiente natural em um determinado espaço, este tipo de análise serve como indicador ambiental para a realização do planejamento territorial e da gestão dos recursos hídricos subterrâneos.

Em hidrogeologia, o conceito de vulnerabilidade das águas subterrâneas começou a ser amplamente utilizado por autores como Aller et al., (1987), Bachmat e Collin, (1987), Foster e Hirata (1988) e Civita (1990). Para Foster et al., (2006, p.16) embora se referisse à suscetibilidade relativa dos aquíferos à contaminação antropogênica, o termo vulnerabilidade inicialmente era usado sem nenhum objetivo de definição formal.

A vulnerabilidade das águas subterrâneas à contaminação pode ser definida em função de um conjunto de características físicas, químicas e biológicas da zona não saturada e/ou do aquitardo confinante que, juntas, controlam a chegada do contaminante ao aquífero (FOSTER et al., 2013). A vulnerabilidade é entendida, portanto, como a suscetibilidade natural dos aquíferos de serem afetados por cargas contaminantes, colocando em risco a qualidade natural das águas subterrâneas (HIRATA; FERNANDES, 2008).

Para Foster et al., (2006, p.4), a forma com que os aquíferos se tornam contaminados ocorre pela entrada das águas através da infiltração direta ou indireta, na superfície do solo ou rocha. As atividades que ocorrem na superfície podem ameaçar a qualidade da água subterrânea, sendo que o solo e as formações rochosas existentes na região, possuem uma determinada capacidade de atenuação natural. Os aquíferos não confinados (livres) são alvo de grande preocupação em relação a sua contaminação, principalmente, onde o nível da água é muito superficial (zona vadosa pouco espessa), apresentando-se demasiadamente expostos às atividades antrópicas existentes em superfície.

O sistema de avaliação de vulnerabilidade aquífera GOD (G - grau de confinamento da água subterrânea; O ocorrência de estratos de cobertura; D - distância até o lençol freático), descrito por Foster et al. (2006, p.23), vem sendo amplamente utilizado em estudos da vulnerabilidade de aquíferos devido à sua simplicidade conceitual e facilidade de aplicação. No Estado do Rio Grande do Sul, alguns trabalhos foram localmente realizados utilizando este sistema, dentre os quais cita-se Camponogara (2006), Descovi Filho (2009), Ertel et al. (2012), Frantz (2005), Löbler et al. (2016), Pavão (2004). Contudo, o objetivo desses estudos não era avaliar modelos de interpolação dos índices vulnerabilidade, sendo empregados diversas técnicas para este fim.

Diferentes interpoladores vêm sendo utilizados para a construção de mapas de vulnerabilidade aquífera em bacias hidrográficas e nem sempre os dados são avaliados a partir de critérios geoestatísticos. O correto mapeamento das variáveis de vulnerabilidade é de fundamental importância para o setor hidroambiental, com destaque para ações associadas à implantação e licenciamento de novos empreendimentos potencialmente poluidores.

Dessa forma, esse estudo busca analisar o uso de diferentes modelos de interpolação de dados do índice de vulnerabilidade aquífera, obtidas pelo sistema GOD, para realizar o mapeamento da suscetibilidade natural à contaminação da água subterrânea na Bacia do Rio Santa Maria. Os resultados contribuirão para adoção do melhor modelo e consequentemente para a proteção mais eficaz dos mananciais da bacia hidrográfica quanto à contaminação ambiental da água subterrânea.

Tendo em vista que a água subterrânea constitui um bem essencial para o abastecimento de água em diversas localidades no meio urbano e rural e, desempenha papel fundamental para o bem-estar dos seres humanos e de muitos ecossistemas aquáticos, este trabalho justifica-se pela importância de obter subsídios para dar suporte à tomada de decisão por parte do poder público e do comitê de gerenciamento da bacia hidrográfica do rio Santa Maria, podendo servir de referência para outras pesquisas e trabalhos na linha de vulnerabilidade das águas subterrâneas.

\section{MATERIAL E MÉTODOS}

\section{Localização da área de estudo}

A Bacia Hidrográfica do Rio Santa Maria (BHRSM) situa-se na fronteira sudoeste do Estado do Rio Grande do Sul (Figura 1), abrangendo uma área de aproximadamente 15.797 $\mathrm{km}^{2}$. Na bacia, localiza-se parte de seis municípios gaúchos sendo: Cacequi, Rosário do Sul, São Gabriel, Santana do Livramento, Lavras do Sul e Dom Pedrito. A população total dos municípios que compõem a BHRSM é de 242.697 habitantes IBGE (2010).

Figura 1. Localização da Bacia Hidrográfica do Rio Santa Maria no Estado do Rio Grande do Sul.

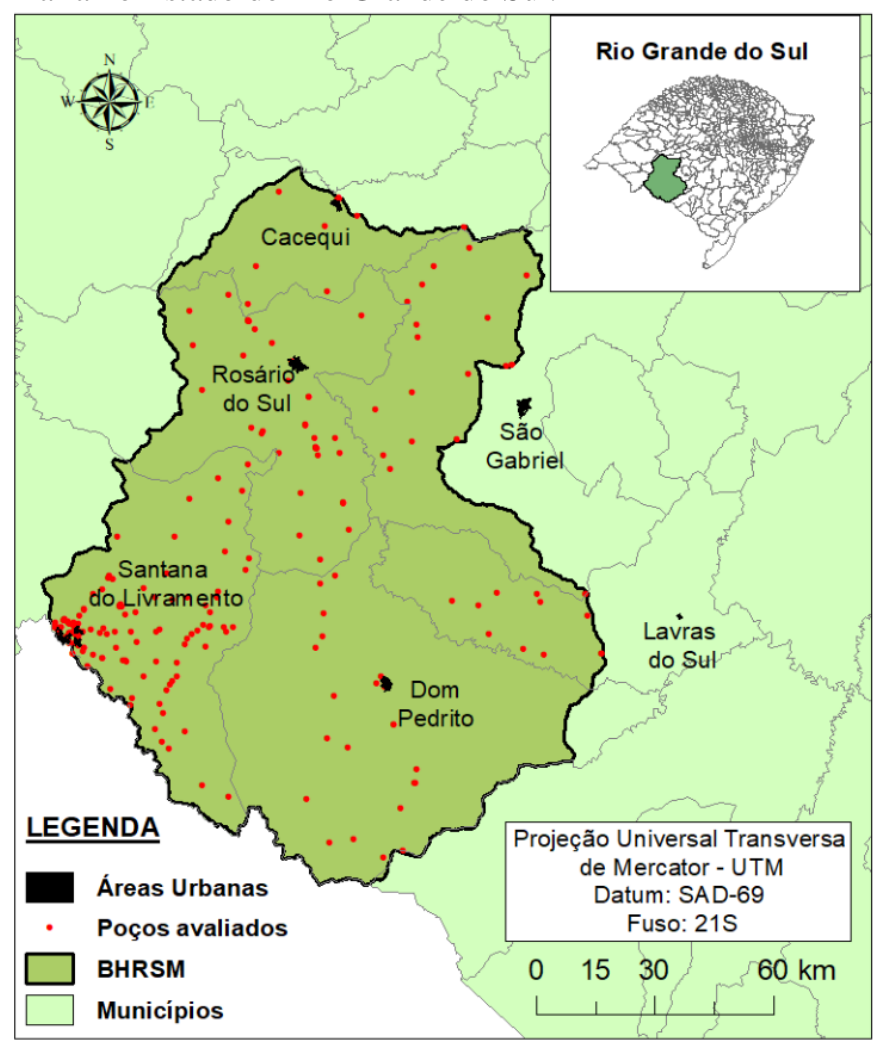

Fonte: CPRM (2016) e Descovi Filho (2009).

A área em estudo está localizada sobre a região de transição entre o Escudo Cristalino (Escudo Cristalino Uruguaio-Sul-Riograndense), a leste, e a Depressão Central, em sua porção central, e ainda, o Planalto da Serra Geral, no extremo oeste (CARRARO, 1974). Essa bacia apresenta 
importância hidrogeológica significativa uma vez que possui, em alguns locais, espessuras sedimentares expressivas, conferindo elevado potencial de armazenamento hídrico subterrâneo.

As rochas dominantes são as sedimentares do tipo arenitos, pertencentes às formações Botucatu, Guará, Sanga do Cabral e Pirambóia (CPRM, 2007). Nesses arenitos, localiza-se o pacote hidrogeológico conhecido internacionalmente como Sistema Aquífero Guarani (SAG), de dimensões continentais. Esse sistema aquífero ocupa parte de oito estados brasileiros (RS, SC, PR, SP, MS, MT e GO), bem como parte do Uruguai, da Argentina e do Paraguai (OEA, 2009).

\section{Métodos utilizados}

Para realização do estudo, utilizou-se informações dos perfis hidrogeológicos de 255 poços tubulares de captações subterrâneas inseridos na BHRSM. Assim, calculou-se o índice GOD para cada poço tubular a partir da metodologia proposta por Foster et al. (2006). Essa metodologia é composta basicamente por 4 fases para o cálculo do Índice de Vulnerabilidade (IV). Fase 1: Determinação do grau de confinamento hidráulico (G), elaborado a partir de informações do tipo do substrato sobrejacente ao aquífero.
Fase 2: O tipo de ocorrência litológica $(\mathrm{O})$, obtida com base nas características do estrato de cobertura de cada poço. Fase 3: Avalia-se o nível estático ou distância do nível da água até a superfície (D), que representa o valor em metros entre a camada da superfície do terreno e o topo do lençol freático do aquífero (altura da zona vadosa).

Por fim, na Fase 4, calcula-se o produto das três fases anteriores, obtendo o IV pela Equação 1.

$$
\mathrm{IV}=\mathrm{G} \cdot \mathrm{O} \cdot \mathrm{D}
$$

Para este estudo, as informações para geração das notas para cada fase do método foram obtidas por meio do Sistema de Informações de Águas Subterrâneas (SIAGAS) (CPRM, 2016) e a partir de coletas realizadas em campo (DESCOVI FILHO, 2009).

Dessa forma, o IV é expresso em intervalo de 0 a 1 , onde valores até 0,1 são considerados insignificantes, de 0,1 a 0,3 são considerados como de baixa vulnerabilidade, 0,3 a 0,5 média vulnerabilidade, 0,5 a 0,7 alta vulnerabilidade e valores maiores que 0,7 são considerados como vulnerabilidade extrema, com alto perigo para contaminação aquífera. A sintetização da metodologia proposta por Foster et al. (2006) é apresentada na Figura 2.

Figura 2. Sistemática das 4 fases para obtenção da vulnerabilidade à contaminação em cada poço tubular.

\section{$1^{\text {a }}$ FASE: GRAU DE CONFINAMENTO DA ÁGUA SUBTERRÂNEA}

\begin{tabular}{|c|c|c|c|c|c|}
\hline Ausência & $\begin{array}{l}\text { Confinado } \\
\text { artesiano }\end{array}$ & Confinado & Semiconfinado & $\begin{array}{l}\text { Năo confinado } \\
\text { (coberto) }\end{array}$ & Năo confinado \\
\hline & & & & & \\
\hline & & 0,2 & 0,4 & 0,6 & 1,0 \\
\hline
\end{tabular}

$2^{\text {a }}$ FASE: OCORRÊNCIA DE ESTRATOS DE COBERTURA (características litológicas e grau de consolidação da zona não saturada)

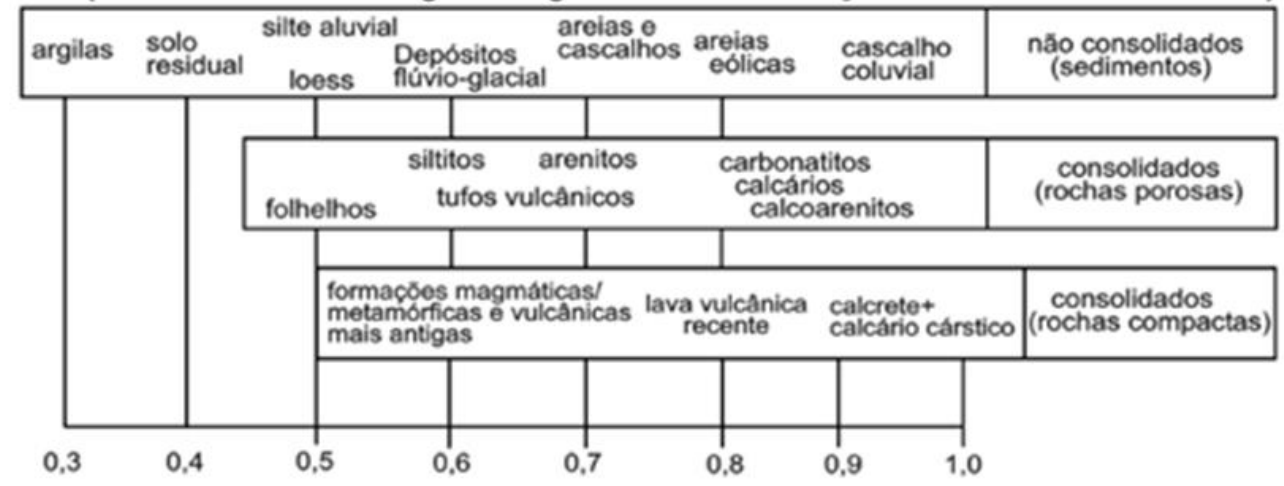

3a FASE: DISTÂNCIA DA ÁGUA SUBTERRÂNEA À SUPERFÍCIE DO TERRENO

\begin{tabular}{|c|c|c|c|}
\hline$>50 \mathrm{~m}$ & $20-50 \mathrm{~m}$ & $5-20 \mathrm{~m}$ & $<5 \mathrm{~m}$ \\
\hline & 1 & 1 & 1 \\
0.4 & 0.6 & 0.8 & 1,0
\end{tabular}

\section{$4^{\mathrm{a}}$ FASE: INDICE DE VULNERABILIDADE}

(produto das fases 1,2 e 3 )

\begin{tabular}{|c|c|c|c|c|c|c|c|c|c|}
\hline$T$ & $T$ & $T$ & $T$ & $T$ & T & $\mathrm{T}$ & $T$ & $T$ & 7 \\
\hline 0,0 & 0,2 & 0,3 & 0,4 & 0.5 & 0,6 & 0.7 & 0,8 & 0,9 & 1.0 \\
\hline Insignificante & Baixo & & Médio & & Alto & \multicolumn{3}{|c|}{ Extremo } & \\
\hline
\end{tabular}

Fonte: Foster et al. (2006) adaptado por Tavares et al. (2009).

Dessa forma, analisaram-se as condições geológicas e hidráulicas de cada poço tubular a partir dos seus perfis geológicos e informações do nível estático, obtendo-se 255 índices de vulnerabilidade avaliados na bacia hidrográfica. De posse desses dados, realizou-se testes para diferentes modelos de interpolação para estes índices. Para essa análise, utilizou- 
se o código livre de programação R (R CORE TEAM, 2017) e o pacote geoestatístico geoR (RIBEIRO JUNIOR; DIGGLE, 2001).

Os gráficos de semivariograma foram obtidos a partir dos IV calculados e da localização geográfica em coordenadas do tipo Universal Transversa de Mercator (UTM), zona 21 do hemisfério sul. O estimador da função de semivariância foi proposto inicialmente por Matheron (1963) e tem a finalidade de fornecer informações sobre a continuidade espacial, ou seja, representa a associação espacial dos dados em que uma função aleatória $\mathrm{Z}(\mathrm{x})$ na direção de um vetor $h$. O estimador do semivariograma $(\gamma)$ é calculado conforme a Equação 2.

$$
\gamma(h)=\frac{1}{2 N(h)} \sum_{\alpha=1}^{N(h)}\left[Z\left(x_{\alpha}\right)-Z\left(x_{\alpha}+h\right)\right]^{2}
$$

Em que N(h) é o número de pares de pontos para cada valor de $\mathrm{h}$, sendo o $\mathrm{Z}(\mathrm{x})$ a variável aleatória regionalizada. Assim, o semivariograma representa a semivariância da diferença da propriedade $\mathrm{Z}$ calculada em pares de pontos separados por $\mathrm{h}$, onde os pares de pontos são $\left(\mathrm{x}_{\alpha}\right)$ e $\left(\mathrm{x}_{\alpha}+\mathrm{h}\right)$.

Esta função é muito utilizada na geoestatística para determinar os padrões ou a estrutura dos dados, sendo, dos métodos estatísticos, o que contém informação mais valiosa sobre a continuidade espacial de determinado atributo. (SOARES, 2006).

A partir desta função, que representa a continuidade espacial dos dados, aplicou-se os seguintes modelos teóricos matemáticos: esférico, exponencial, gaussiano e linear. O intuito foi avaliar o melhor modelo para a interpolação dos dados e obter uma interpolação com melhor qualidade. Para avaliação do modelo mais adequado, considerou-se o método dos mínimos quadrados (MMQ) como estimador de ajuste. Esse estimador busca a otimização entre os dados e o modelo teórico matemático, procurando encontrar o melhor ajuste para um determinado conjunto de dados, minimizando a soma dos quadrados das diferenças entre o valor estimado e os dados observados.

\section{RESULTADOS E DISCUSSÃO}

A partir dos dados inventariados e de posse de um banco de dados hidrogeológicos com informações dos perfis geológicos dos poços tubulares, procedeu-se com a metodologia GOD para obtenção do IV em cada poço. Em um primeiro momento, estabeleceu-se o grau de confinamento hidráulico (G), onde foram identificados 191 poços em condições de confinamento livre, ou seja, a superfície freática nesses pontos está em contato direto com a pressão atmosférica local por meio dos poros das rochas areníticas. Essa característica indica menor proteção natural à poluição, uma vez que essa característica hidráulica permite a percolação de possíveis contaminantes devido à ação da gravidade. Os poços restantes foram considerados semiconfinados e confinados (artesianos), recebendo notas mais baixas, pois teoricamente possuem mais proteção natural em virtude do seu confinamento (presença de camadas impermeáveis que dificultam e/ou impedem o processo de infiltração) e, por isso, são menos vulneráveis (FOSTER et al., 2006).

$\mathrm{Na}$ ocorrência litológica (O) (obtida com base no perfil geológico de cada poço), foi identificado que grande parte do total avaliado (217 poços) encontra-se em geologias sedimentares das formações Botucatu e Guará, recebendo pesos entre 0,6 a 0,8. Essas formações são compostas principalmente por rochas areníticas que, na área de estudo, são porosas e com elevada condutividade hidráulica, o que facilita a circulação/transmissão da água e a percolação de potenciais contaminantes e, consequentemente, apresentam aspectos de maior risco de contaminação

Os demais poços avaliados receberam pesos menores por pertencerem à formações geológicas propensas a possuir maior resistência natural à contaminação, caso das rochas da formação Serra Geral. Essas formações são compostas por derrames de basaltos, andesitos, riodacitos e riolitos, de filiação toleítica, onde intercalam-se arenitos intertrápicos na base e sedimentos vulcanogênicos (CPRM, 2007).

Os pesos atribuídos ao nível estático (distância do nível da água até a superfície) variaram entre 0,4 a 1, uma vez que houve variação entre zero (nível da água subterrânea e do terreno iguais) e 84 metros.

Dessa forma, calculou-se o IV de 255 poços tubulares localizados na BHRSM, sendo identificadas todas as classes de vulnerabilidade na bacia. Do total, houve uma predominância da classe de alta vulnerabilidade em 47,45\% dos pontos avaliados (121 poços), seguidos da classe de média vulnerabilidade em $23,14 \%$ (59 poços). A classe de baixa vulnerabilidade apresentou um percentual de 14,50\% (37 poços) e a classe insignificante perfez 7,05\% (18 poços). Por sua vez, a classe de extrema vulnerabilidade aquífera apresentou 7,85\% (20 poços). O número de observações realizadas foi sistematizado no histograma de frequência da Figura 3.

Figura 3. Histograma de frequência dos cálculos de IV em 255 poços tubulares na Bacia Hidrográfica do Rio Santa Maria no Estado do Rio Grande do Sul.

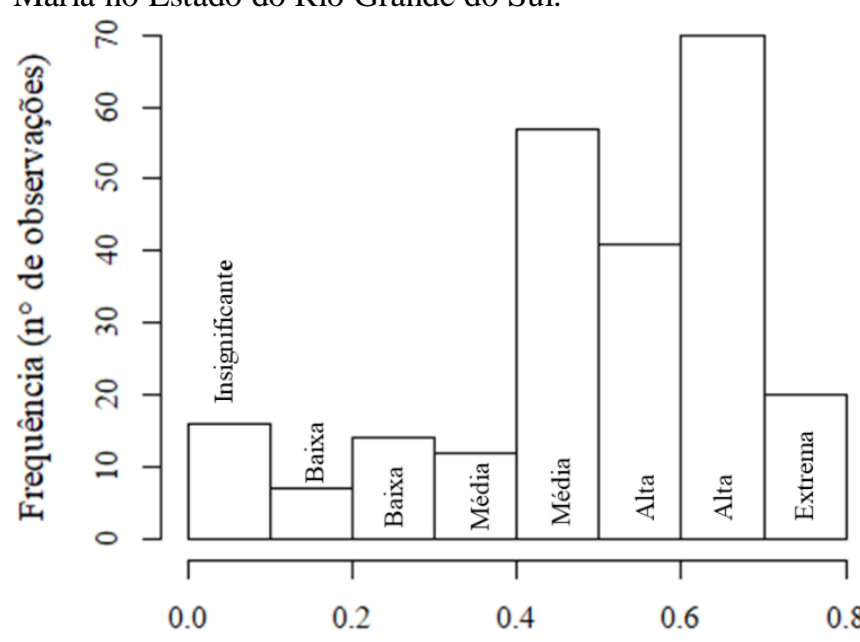

Índice de Vulnerabilidade

No histograma de frequência da Figura 3, observa-se de forma sucinta, o universo de dados de IV avaliados obtidos pelo método GOD, com destaque para as classes de alta vulnerabilidade (IV entre 0,5 a 0,7 ) e média vulnerabilidade aquífera (IV entre 0,3 a 0,5). No total, os valores dos índices de vulnerabilidade para a BHRSM variaram entre 0,08 (insignificante) a 0,72 (extrema vulnerabilidade).

Em geral, a predominância da classe de alta vulnerabilidade aquífera obtida pelo sistema GOD deu-se em função dos tipos litológicos encontrados na bacia (rochas 
sedimentares com predominância de arenitos, siltitos, argilitos e depósitos aluviais). Outro fator preponderante foi a distância da superfície do terreno ao lençol freático que, apesar da alta variabilidade, teve 71 poços onde o nível estático foi menor que 5 metros e 114 poços onde o nível situou-se entre 5 a 20 metros, recebendo maiores pesos neste quesito.

Assim, a partir desses dados e das localizações em coordenadas UTM, iniciou-se a análise estrutural, aplicando a função de semivariograma e verificando-se os ajustes dos diferentes modelos teóricos de interpolação. Na Figura 4 observa-se a aplicação dos seguintes modelos teóricos nos gráficos de semivariograma: esférico (a), exponencial (b), gaussiano (c) e linear (d).
A partir da semivariância, verifica-se a plotagem dos modelos teóricos matemáticos (linha contínua vermelha) de interpolação nos dados obtidos pelo método GOD (Figura 4). Além dos modelos teóricos, são apresentados os parâmetros do semivariograma, que são variáveis de ajuste para posterior aplicação da krigagem. Esses parâmetros são: o efeito pepita, que é o valor da função de semivariograma na origem; o patamar, que indica o ponto no eixo y; e o alcance, que corresponde à distância no eixo $\mathrm{x}$, a partir da qual as amostras se tornam independentes.

É possível avaliar em primeiro momento que, em geral, os modelos teóricos obtiveram boa aderência aos pontos plotados no gráfico, com exceção do modelo linear.

Figura 4. Semivariograma dos dados de IV, calculados pelo método GOD e, localização geográfica plotados. A linha contínua em cor vermelha representa o modelo teórico matemático que descreve a distribuição espacial.

Modelo Esférico

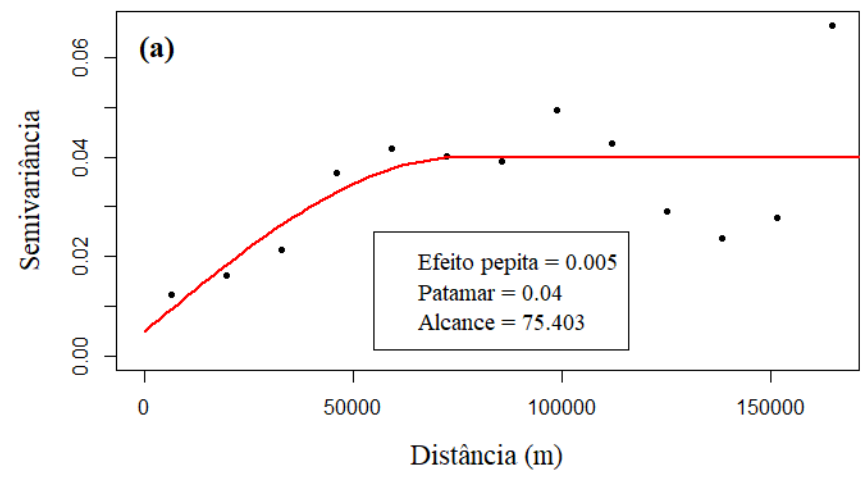

Modelo Gaussiano

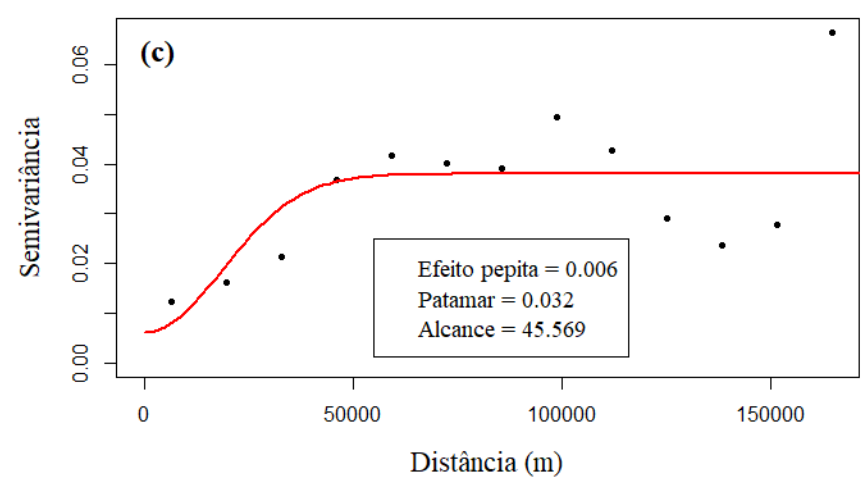

O modelo linear demonstrou-se ineficiente para a interpolação desses dados, uma vez que não apresentou tendência, com patamar zero e alcance infinito. Os demais modelos apresentaram um bom ajuste visual, evidenciadas pelas tendências demonstradas pelos pontos plotados de IV e localização geográfica nos gráficos de semivariância.

No entanto, visualmente não é possível afirmar qual modelo apresenta menores erros de interpolação pela krigagem, sendo necessário a aplicação de estimadores de erros que levem em consideração a variável medida e a variável modelada. Dessa forma, aplicou-se o Método dos Mínimos Quadrados (MMQ) com o intuito de observar numericamente essa diferença, com os resultados apresentados na Tabela 1.

Observa-se então que o modelo esférico apresentou o menor MMQ, demonstrando-se como o mais eficiente para a interpolação desses dados, ao contrário do modelo linear, que
Modelo Exponencial
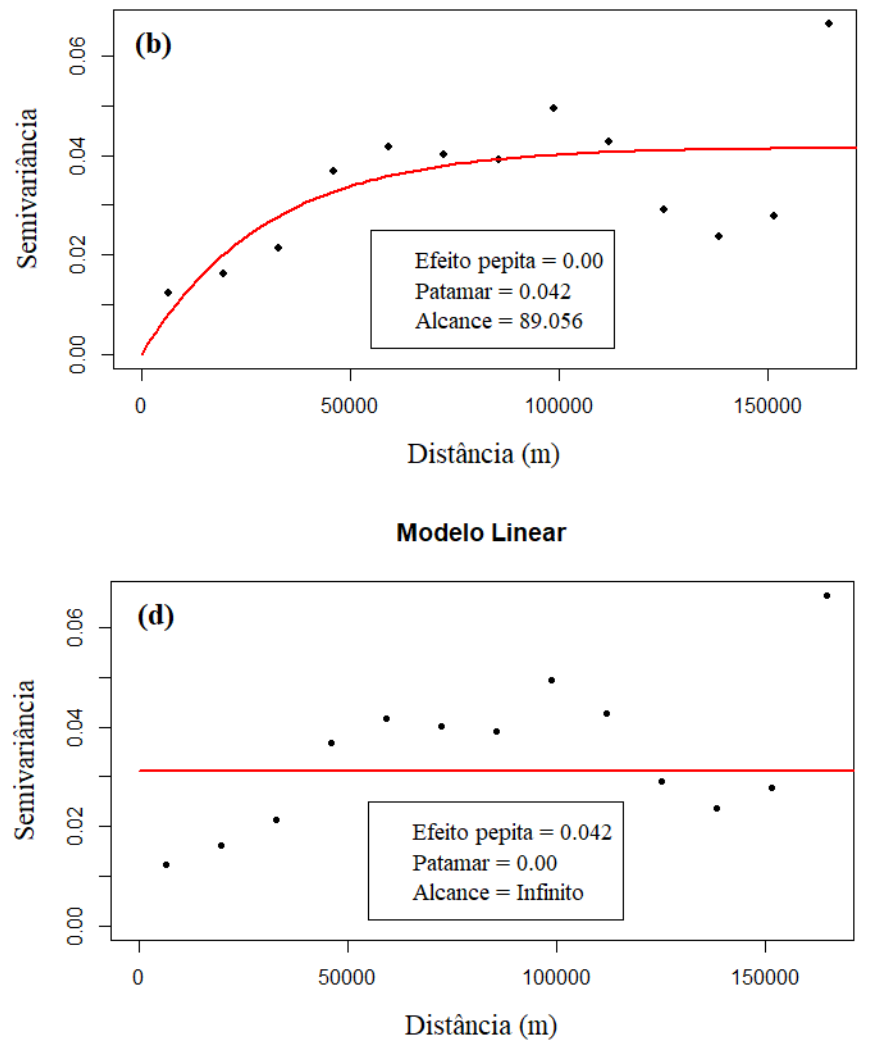

apresentou maior MMQ, sendo considerado, portanto, o modelo menos indicado para interpolar esses dados. Os modelos exponencial e gaussiano apresentaram MMQ próximos a 1, que podem ser considerados razoáveis para interpolação e sua utilização não é inteiramente errônea. Essa afirmação pode ser constatada pela boa aderência ao conjunto de dados observados anteriormente na Figura 4.

Tabela 1. Cálculo do Método dos Mínimos Quadrados (MMQ) obtidos a partir dos gráficos de semivariograma.

\begin{tabular}{lc}
\hline Modelo & MMQ \\
\hline Esférico & 0,83 \\
Exponencial & 1,08 \\
Gaussiano & 1,12 \\
Linear & 4,16 \\
\hline
\end{tabular}


Neste contexto, observou-se que o modelo esférico é o mais indicado, pois oferece menores erros ao estimar a vulnerabilidade aquífera na bacia hidrográfica. Com a finalidade de observar a distribuição espacial da vulnerabilidade na bacia, interpolou-se os dados de IV pelo método da krigagem a partir do modelo esférico, utilizando-se os parâmetros de ajuste (efeito pepita, patamar e alcance). Segue abaixo na Figura 5 os resultados obtidos pela interpolação na BHRSM.

Figura 5. Vulnerabilidade da Bacia Hidrográfica do Rio Santa Maria interpolado por krigagem, utilizando o modelo esférico e os parâmetros de ajuste.

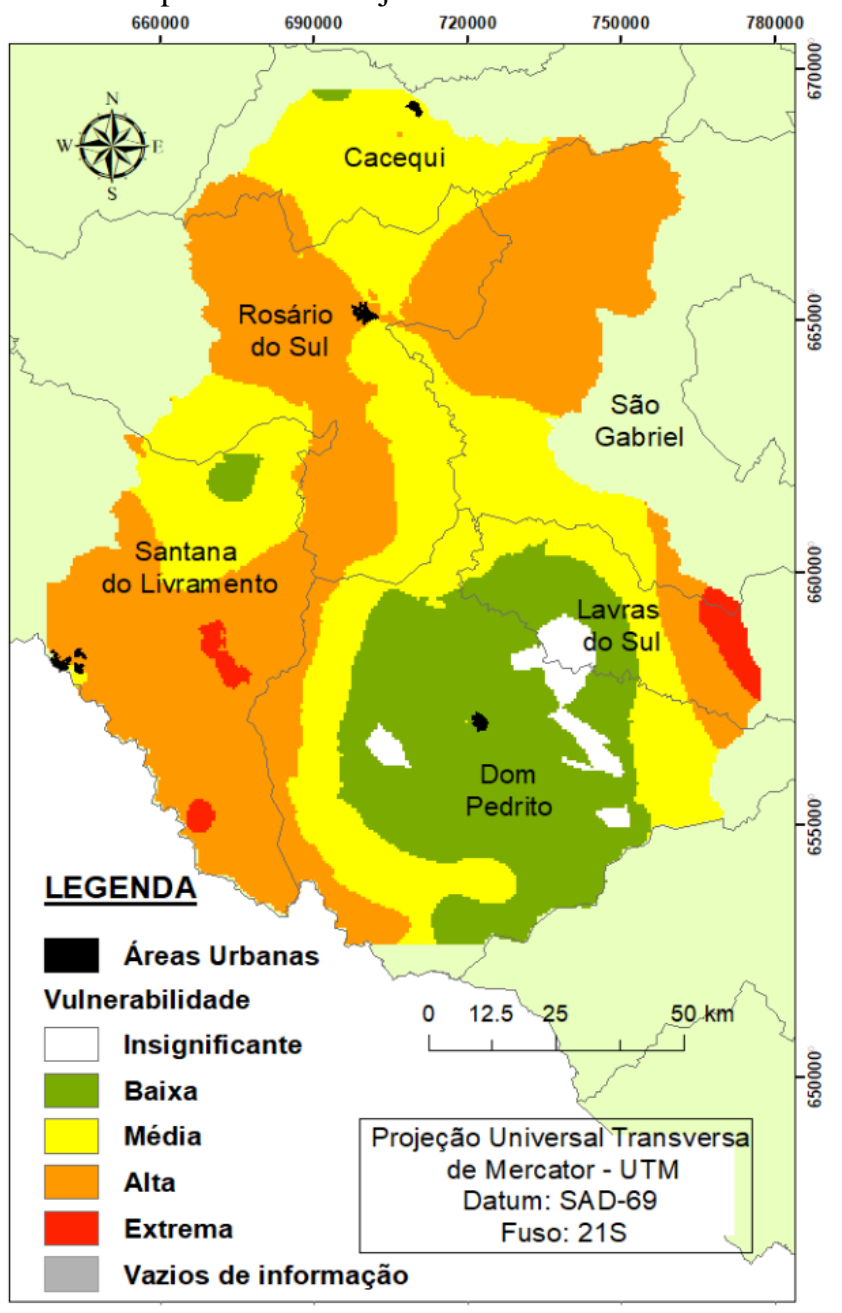

A partir da interpolação pelo método de krigagem com o modelo esférico, observou-se a distribuição espacial dos dados da vulnerabilidade aquífera obtida pelo método de Foster et al., (2006). A classe de extrema vulnerabilidade aquífera foi constatada em alguns pontos da bacia nos municípios de Santana do Livramento e Lavras do Sul. Contudo, não foi identificada extrema vulnerabilidade pelo método próximo das áreas urbanas desses municípios.

Para a classe de alta vulnerabilidade, notou-se um amplo predomínio na bacia, principalmente na porção sudeste do município de Santana do Livramento a sudoeste de Dom Pedrito, porção central, sudeste e nordeste de Rosário do Sul, noroeste de São Gabriel e central de Lavras do Sul.

Comparando-se os resultados com a pesquisa realizada por Descovi Filho (2009), o autor encontrou as classes de vulnerabilidade média e alta como predominantes, equivalentes respectivamente a 30,2\% e 35,6\% da BHRSM. Löbler et al. (2016), definiram um percentual de 20,56\% da classe de média vulnerabilidade e $56,56 \%$ como alta vulnerabilidade nos poços avaliados.

É possível inferir que a análise geoestatística da interpolação de dados possui importância significativa quando se trabalha com bacias consideradas de grandes dimensões. A escala do mapa é, portanto, muito relevante para a correta escolha do interpolador.

Algumas áreas da bacia apresentaram pontos com vazios de informações (cor cinza) onde não foi possível realizar a krigagem. Salienta-se que esses pontos são mínimos (cerca de $1,1 \%$ ), sendo desconsiderados na análise.

Foi identificada alta vulnerabilidade aquífera nas áreas urbanas dos municípios de Santana do Livramento e Rosário do Sul, constituindo um risco maior de contaminação para a água subterrânea nessas localidades, uma vez que a zona urbana constitui um risco potencial de contaminação devido à produção de efluentes industriais e domésticos.

As áreas em que houveram predomínio da classe de extrema e alta vulnerabilidade, merecem especial atenção no que tange ao monitoramento e ao licenciamento de possíveis novos empreendimentos que possam ser fonte de poluição para os aquíferos. Nessas áreas, sudeste de Santana do Livramento, central de Lavras do Sul e sul de São Gabriel é importante que se acompanhe a possível instalação de empreendimentos potencialmente poluidores e que haja restrições em legislações, além de uma fiscalização ambiental eficiente, a fim de garantir a proteção e o manejo adequado dos mananciais de água subterrânea.

É importante salientar que o sistema GOD possui algumas limitações, que são inerentes a qualquer modelo teórico da realidade. De acordo com Foster et al. (2006, p.27) a limitação do método pode ser devido à presença de corpos de água superficiais indefinidos (permanentes ou intermitentes), a exploração excessiva do aquífero, que pode variar a profundidade do lençol freático e mesmo o grau de confinamento do aquífero. Outro fator limitante importante é a possibilidade de ocorrência de grandes alinhamentos e de fraturas em formações basálticas da formação Serra Geral, gerando incertezas quanto à magnitude dos componentes de fluxo preferencial.

Os mapas de vulnerabilidade são importantes para avaliar o perigo de contaminação da água subterrânea associado às descargas de substâncias que ocorrem na superfície do terreno. O método foi desenvolvido para análise da vulnerabilidade intrínseca, portanto, não contempla a detecção ou monitoramento de possíveis potenciais contaminantes abaixo do subsolo, como por exemplo em casos de vazamentos de tanques de armazenagem subterrâneos, lixiviação de aterros de resíduos sólidos sob a camada de solo ou descargas efluentes em pedreiras e minerações.

\section{CONCLUSÕES}

O método de krigagem aplicado com o modelo esférico apresentou o melhor ajuste para interpolar os dados de vulnerabilidade aquífera pelo método GOD na Bacia Hidrográfica do Rio Santa Maria. O modelo esférico ofereceu menores erros ao estimar a vulnerabilidade aquífera na bacia hidrográfica. 
A elaboração do mapa da vulnerabilidade demonstra áreas onde a água subterrânea está naturalmente sujeita a maiores riscos de contaminação, devido às suas características hidrogeológicas.

A porção sudeste do município de Santana do Livramento, a porção central de Rosário do Sul, noroeste de São Gabriel e leste de Lavras do Sul são passíveis de ações de conservação por parte do poder público, a fim de manter a qualidade da água dos mananciais subterrâneos nessas localidades.

\section{REFERÊNCIAS}

ALLER, L.; LEHR, J. H.; PETTY, R.; BENNETT, T. Drastic: A standardized system for evaluating groundwater pollution potencial using hydrogeologic setting. National Water Well Association, Ohio. p.38-57, 1987.

BACHMAT, Y.; COLLIN, M. Mapping to assess groundwater vulnerability to pollution. In: Vulnerability of soil and groundwater to pollutants. Proceedings and Information. p.297-307, 1987.

CAMPONOGARA, I. Vulnerabilidade natural do Sistema Aquífero Guarani, em Santana do Livramento, RS, com uso de geotecnologias. 2006. 110f. Dissertação (Mestrado em Geomática) - Universidade Federal de Santa Maria, Santa Maria, 2006.

CARRARO, C. C. Mapa Geomorfológico do Estado do Rio Grande do Sul. FAPERGS - UFRGS/Instituto de Geociências. 1974, 1: 1.000.000.

CIVITA, M. Assesment of aquifer vulnerability to contamination. Protezione e Gestione delle Acque Sotteranee: Mettodologie, Technologie e Obbietivi. Marano sul Panaro, v. 3, p. 39-86, 1990.

CPRM. Companhia de Pesquisa em Recursos Minerais. Sistema de Informações de Águas Subterrâneas. 2016. Banco de dados dos poços cadastrados. Disponível em: <http://siagasweb.cprm.gov.br>. Acesso em abril de 2016.

CPRM. Companhia de Pesquisa em Recursos Minerais. 2007. Mapa Geológico do Estado do Rio Grande do Sul. Escala de $1: 750.000$.

DESCOVI FILHO, L. L. V. Subsídios ambientais para a gestão das águas subterrâneas na bacia hidrográfica do rio Santa Maria. 2009. 156f. Dissertação (Mestrado em Engenharia Civil) - Universidade Federal de Santa Maria, Santa Maria, 2009.

ERTEL, T.; LÖBLER, C. A.; SILVÉRIO DA SILVA, J. L. Índice de vulnerabilidade das águas subterrâneas no município de Rosário do Sul, Rio Grande do Sul. Revista Eletrônica em Gestão, Educação e Tecnologia Ambiental, Santa Maria, v.7 n.7, p.1400-1408, 2012.

FOSTER, S. S. D.; HIRATA, R. Groundwater pollution risk assessment. A methodology using available data. Pan American Center for Sanitary Engineering. and Environmental Sciences. Lima, 1988. 87p.
FOSTER, S.; HIRATA, R. C. A.; GOMES, D.; D’ELIA, M.; PARIS, M. Proteção da Qualidade da Água Subterrânea: um guia para empresas de abastecimento de água, órgãos municipais e agências ambientais. São Paulo: SERVMAR, 2006. 114p.

FOSTER, S.; HIRATA, R.; ANDREO, B. The aquifer pollution vulnerability concept: aid or impediment in promoting groundwater protection?. Hydrogeology Journal, v.21, n.7, p.1389-1392, 2013.

FRANTZ, L. C. Avaliação do Índice de Vulnerabilidade do Aqüífero Guarani no Perímetro Urbano da Cidade de Santana do Livramento. 2005. 125f. Dissertação (Mestrado em Engenharia Civil) - Universidade Federal de Santa Maria, Santa Maria, 2005.

HIRATA, R.; FERNANDES, A. J. Vulnerabilidade à poluição de aquíferos. In: FEITOSA. F. A. C.; FILHO J. M.; FEITOSA E. C.; DEMÉTRIO J. G. A. (Org.) Hidrogeologia: Conceitos e aplicações. 3 ed. Rio de Janeiro: CPRM; LABHID, 2008. Cap. 5.4, p.406-425.

IBGE. Instituto Brasileiro de Geografia e Estatística. Cidades. $2010 . \quad$ Disponível em: <http://www.ibge.gov.br/cidadesat/default.php> Acesso em 18 de maio de 2016.

LÖBLER, C. A.; BORBA, W. F.; SILVÉRIO DA SILVA, J. L. Vulnerabilidade das águas subterrâneas da bacia do rio Santa Maria, Rio Grande do Sul. Ciência e Natura, Santa Maria, v.38, n.1, p.84-94, 2016.

MATHERON, G. Principles of geostatistics. Economic geology, v.58, n.8, p.1246-1266, 1963.

OEA. Organização dos Estados Americanos. Programa Estratégico de Ação (PEA) do Projeto Sistema Aquífero Guarani (PSAG). 2009. Disponível em: < http://www.ana.gov.br/bibliotecavirtual/arquivos/2010022317 2711_PEA_GUARANI_Port_Esp.pdf>

PAVÃO, A. D. M. Avaliação do índice de vulnerabilidade na bacia hidrográfica do rio Santa Maria. 2004. $107 f$. Dissertação (Mestrado em Engenharia Civil) - Universidade Federal de Santa Maria, Santa Maria, 2004.

R CORE TEAM. R language definition. Vienna, Austria: R foundation for statistical computing, 2017.

RIBEIRO JUNIOR, P. J.; DIGGLE P. J. geoR: a package for geostatistical analysis. R news, v.1, n.2, p.14-18, 2001.

SOARES, A. Geoestatística para as ciências da terra e do ambiente. 2.ed. Lisboa: Instituto Superior Técnico, 2006. 232 p.

TAVARES, P. R. L.; CASTRO, M. A. H. D.; COSTA, C. T. F. D.; SILVEIRA, J. D. G. P. D.; JÚNIOR, F. J. B. D. A. Mapeamento da vulnerabilidade à contaminação das águas subterrâneas localizadas na Bacia Sedimentar do Araripe, Estado do Ceará, Brasil. Revista Escola de Minas, Ouro Preto, v.62, n.2, p.227-236, 2009. 